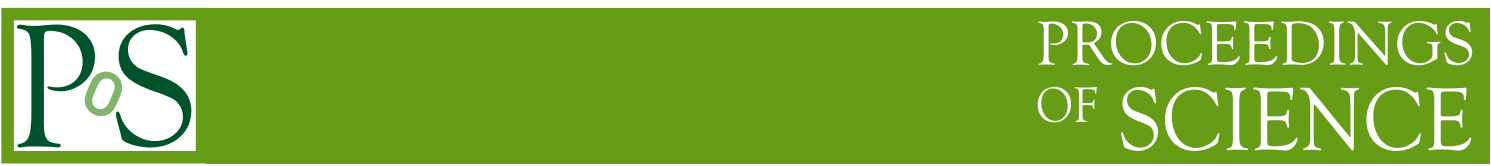

\title{
Studies of mesic atoms and nuclei
}

\author{
Eliahu Friedman, Avraham Gal* \\ Racah Institute of Physics, The Hebrew University, 91904 Jerusalem, Israel \\ E-mail: avragalesavion.huji.ac.il
}

Aleš Cieplý, Jaroslava Hrtánková, Jiří Mareš

Nuclear Physics Institute, 25069 Rez, Czech Republic

\begin{abstract}
$K^{-}$mesons offer a unique setting where mesic atoms have been studied both experimentally and theoretically, thereby placing constraints on the possible existence and properties of meson-nuclear quasibound states. Here we review progress in this field made recently by the Jerusalem-Prague Collaboration using near-threshold $K^{-} N$ scattering amplitudes generated in several meson-baryon coupled channels models inspired by a chiral EFT approach. Our own procedure of handling subthreshold kinematics self consistently is used to transform these freespace energy dependent amplitudes to in-medium density dependent amplitudes from which $\mathrm{K}^{-}$ optical potentials are derived. To fit the world data of kaonic atoms, these single-nucleon optical potentials are augmented by multi-nucleon terms. It is found that only two of the studied models reproduce also the single-nucleon absorption fractions available from old bubble chamber experiments. These two models are then checked for possible $K^{-}$nuclear quasibound states, despite realizing that $K^{-}$optical potentials are not constrained by kaonic atom data at densities exceeding half nuclear-matter density. We find that when such states exist, their widths are invariably above $100 \mathrm{MeV}$, forbiddingly large to allow observation. Multi-nucleon absorption is found to be substantial in this respect. This suggests that observable strongly bound $K^{-}$mesons are limited to the very light systems, such as $K^{-} p p$.
\end{abstract}

XVII International Conference on Hadron Spectroscopy and Structure 25-29 September, 2017

University of Salamanca, Salamanca, Spain

${ }^{*}$ Speaker. 


\section{Introduction and methodology}

Searches for meson $\left(\bar{K}, \eta, \eta^{\prime}, \omega, \phi\right)$-nuclear quasibound states were reviewed in Hadron2017 by Nanova [1]. Here we focus on $K^{-}$mesons for which atomic data provide exclusively useful constraints beyond those available from near-threshold $K^{-} N$ data $[2,3]$. To consider $K^{-}$atoms and quasibound nuclear states we construct $K^{-}$optical potentials based on free-space $K^{-} N$ amplitudes taken from Ref. [4] as shown in Fig. 1. These six amplitude sets exhibit considerable model dependence, particularly below threshold, $E<E_{\text {th }}$, and for $K^{-} n$ also above. We note that near-threshold meson optical potentials require subthreshold meson-nucleon amplitudes for input $[5,6]$.
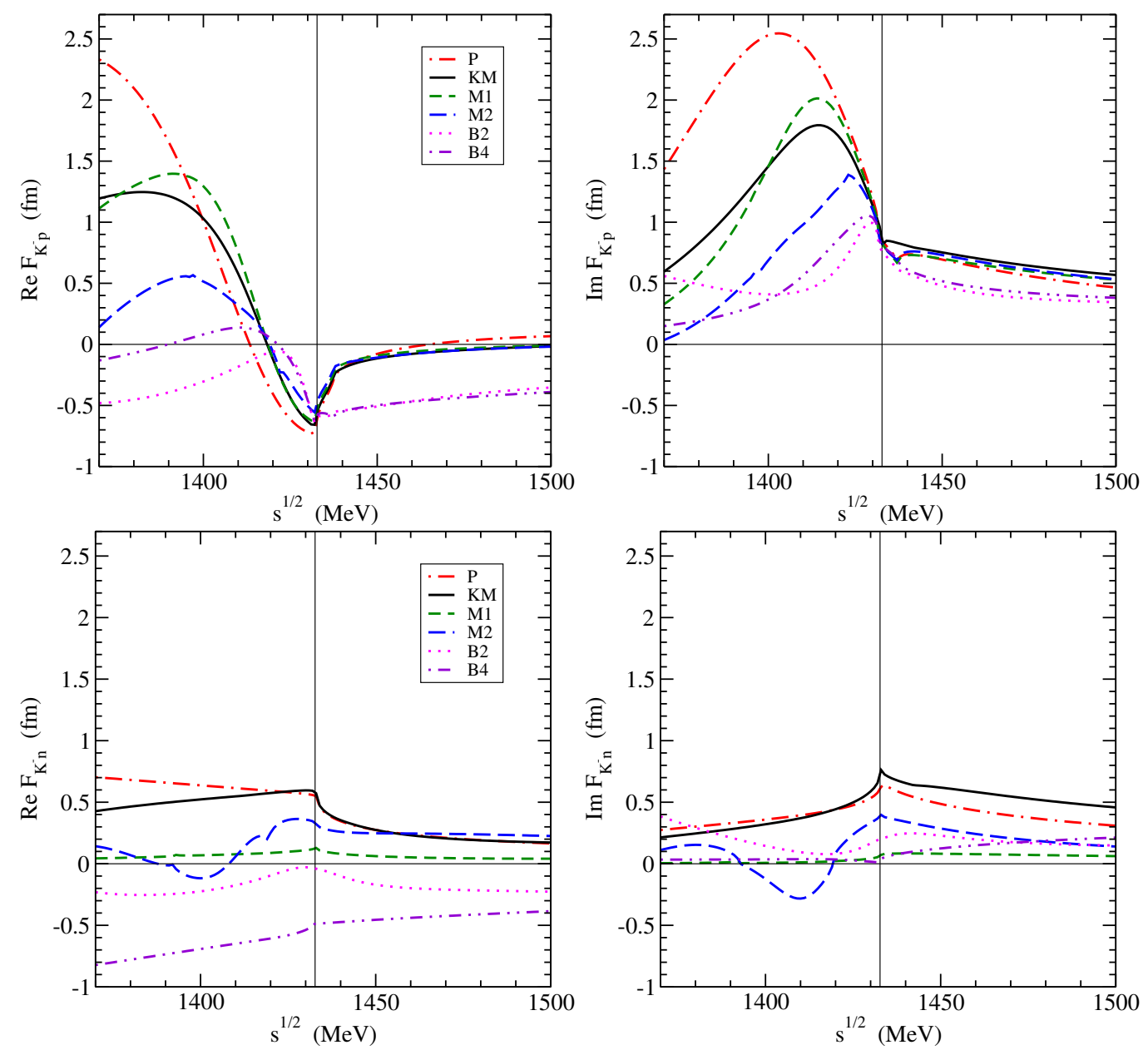

Figure 1: Energy dependence of real (left) and imaginary (right) parts of $K^{-} p$ (top) and $K^{-} n$ (bottom) scattering amplitudes from six meson-baryon coupled-channel chirally inspired EFT models [4] constrained by threshold and low-energy $K^{-} N$ data. Threshold energies $E_{\text {th }}$ are marked by vertical lines.

The free-space amplitudes of Fig. 1 are then modified to account for in-medium effects, the leading one being the Pauli principle. The energy dependence of several versions of in-medium amplitudes is compared in Fig. 2 with that of the underlying free-space Prague (P) model amplitude from Fig. 1. The version applied in our recent calculations [7, 8, 9] is that denoted WRW [10]. 

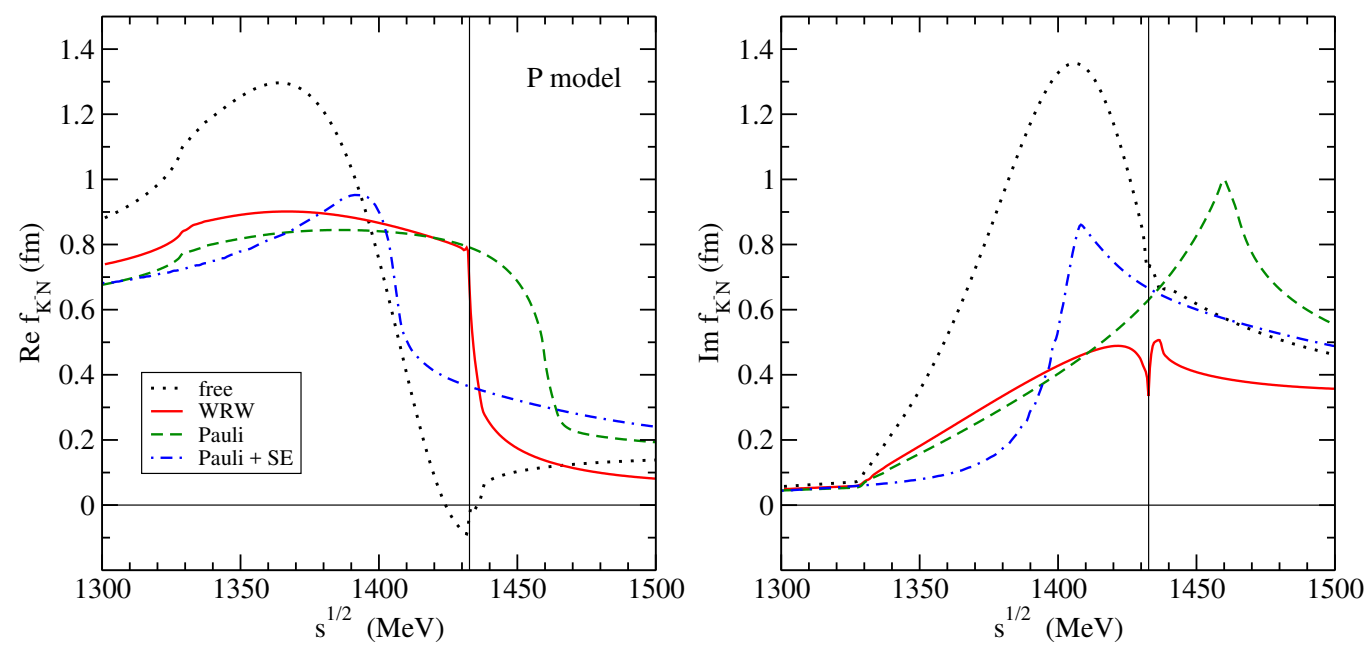

Figure 2: Energy dependence of the free-space (dotted) P-model amplitudes $f_{K^{-} N}=\frac{1}{2}\left(f_{K^{-} p}+f_{K^{-} n}\right)$, where $f(E)=F\left(E ; p=p^{\prime}=0\right)$, and of several versions of in-medium P-model amplitudes at nuclear-matter density $\rho_{0}=0.17 \mathrm{fm}^{-3}$ (left: real parts, right: imaginary parts). Figure adapted from Ref. [9].

The subthreshold energy $\sqrt{s}=E_{\text {th }}+\delta \sqrt{s}$ at which hadron optical potentials $V_{h}(\sqrt{s})$ are to be evaluated is then determined by solving a self-consistency equation $[5,6,7,8,9]$

$$
\delta \sqrt{s}(\rho)=-B_{N} \rho / \bar{\rho}-\beta_{N}\left[T_{N}(\rho / \bar{\rho})^{2 / 3}+B_{h} \rho / \rho_{0}+V_{C}\left(\rho / \rho_{0}\right)^{1 / 3}\right]+\beta_{h} \operatorname{Re} V_{h}(\sqrt{s})
$$

in which $V_{h}(\sqrt{s})=\tilde{t}_{h N}(\sqrt{s}) \rho$ serves as input with $\tilde{t}_{h N}$ denoting an in-medium $h N t$-matrix. Next, $V_{C}$ is the $h$-nucleus Coulomb potential, $\beta_{N(h)}=m_{N(h)} /\left(m_{N}+m_{h}\right), B_{N}=8.5 \mathrm{MeV}, T_{N}=23 \mathrm{MeV}$ and $B_{h}$ is the hadron binding energy obtained by solving the Klein-Gordon equation with potential $V_{h}(\sqrt{s})$. The output $\tilde{t}_{h N}$ and $V_{h}$ have thus become density dependent. Fig. 3 demonstrates a steady decrease of $\delta \sqrt{s}(\rho)$, denoted $E-E_{\mathrm{th}}$, for pionic [11] and kaonic [7] atoms where $B_{h} \approx 0$, reaching comparable values of $\delta \sqrt{s}$ between -20 to $-30 \mathrm{MeV}$ at the nuclear surface $\left(\rho \approx \frac{1}{2} \rho_{0}\right)$.
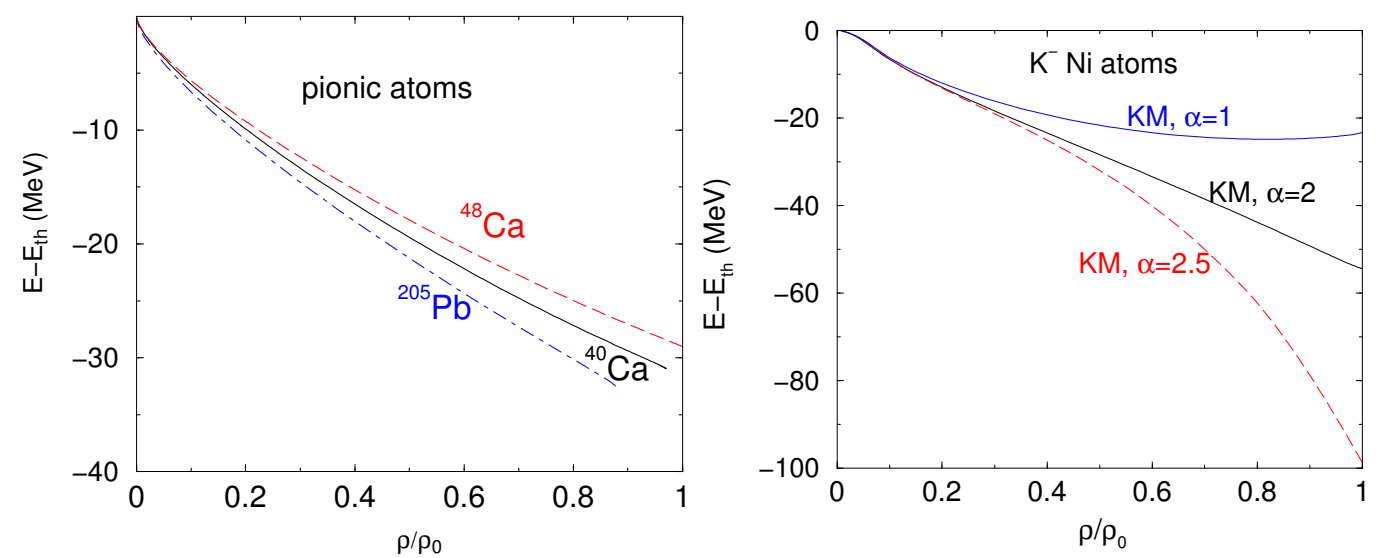

Figure 3: Density dependence of meson-nucleon energy shifts involved in mesic atom calculations. The various $\mathrm{KM} \alpha$ branches of model KM in the right panel are defined in Sect. 2. Figure adapted from Ref. [11]. 


\section{News from kaonic atoms}
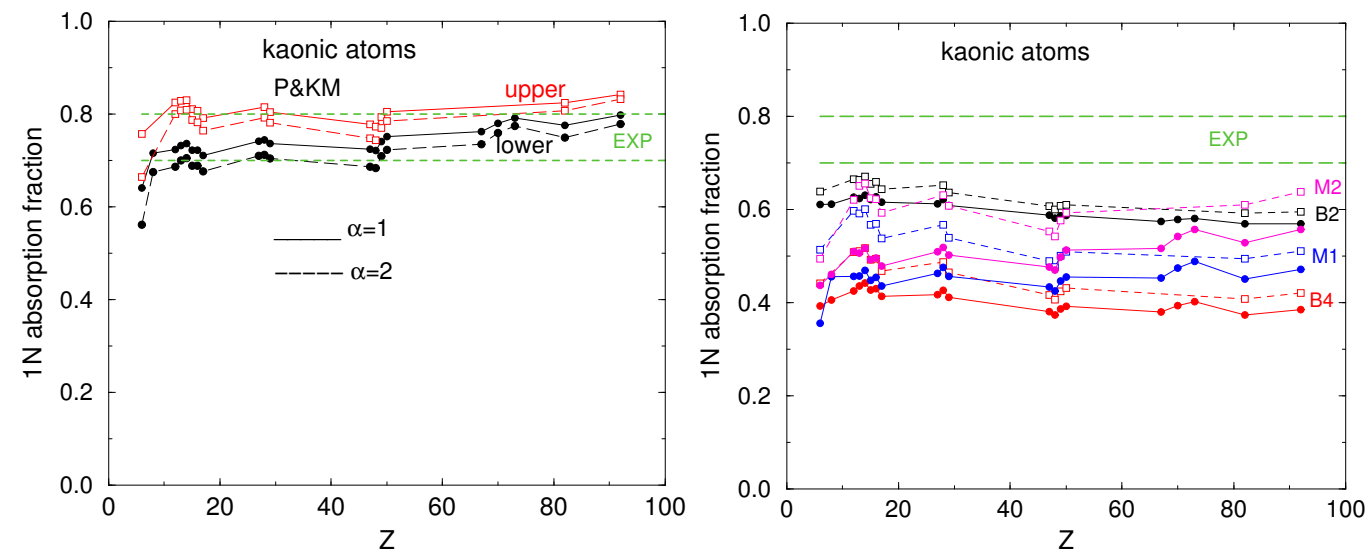

Figure 4: Calculated SNAF [7] in models P \& KM (left) and in other models (right). For P \& KM, solid circles (open squares) stand for 'lower' ('upper') states. For right-panel notations and choice of $\alpha$, see [7].

The single-nucleon $(1 N)$ optical potentials $V_{K^{-}}^{1 N}=\tilde{t}_{h N}(\rho) \rho$ of Sect. 1 , with in-medium $t$-matrices $\tilde{t}_{h N}(\rho)$ constructed from free-space $t$-matrices proportional to the amplitudes $F$ of Fig. 1, fail miserably to fit kaonic atom data. However, adding a phenomenological amplitude $B\left(\rho / \rho_{0}\right)^{\alpha}$ to simulate multi-nucleon $(m N)$ processes and varying the complex strength parameter $B$ upon gridding on the exponent $\alpha$, good fits are obtained in all six cases, comparable in quality to published phenomenological best fits [12]. Calculations are then made for single-nucleon absorption fractions (SNAF) measured long ago in bubble-chamber experiments on nuclear species from $\mathrm{C}$ to $\mathrm{Br}$ (see [7] for references) which are consistent with a common value $\mathrm{SNAF}_{\exp } \approx 0.75 \pm 0.05$. These absorption fractions are computed for any atomic state $\psi_{j}$ by splitting $V_{K^{-}}$to its $1 N$ and $m N$ components, $V_{K^{-}}=V_{K^{-}}^{1 N}+V_{K^{-}}^{m N}$, and evaluating $\Gamma_{1 N}^{j} / \Gamma_{\text {tot }}^{j}$ according to

$$
\Gamma_{\text {tot }}^{j}=\Gamma_{1 N}^{j}+\Gamma_{m N}^{j}: \quad \Gamma_{1 N}^{j} \sim \int\left|r \psi_{j}\right|^{2} \operatorname{Im} V_{K^{-}}^{1 N} d r, \quad \Gamma_{m N}^{j} \sim \int\left|r \psi_{j}\right|^{2} \operatorname{Im} V_{K^{-}}^{m N} d r .
$$

Inspecting the overlap of $\left|r \psi_{j}\right|^{2}$ with $\operatorname{Im} V_{K^{-}}$in the expression for $\Gamma_{\text {tot }}^{j}$ one finds that it generally peaks at $15-20 \%$ of central nuclear density $\rho_{0}$ for 'lower' states and $10-15 \%$ of $\rho_{0}$ for 'upper' states. Fig. 4 shows very good agreement between calculation and experiment for optical potentials based on the $1 N$ input of models $\mathrm{P}$ and $\mathrm{KM}$, including a $m N$ term, and substantial disagreement for the other four models.

Fig. 5 shows $K^{-}$optical potentials ' $\mathrm{KM} \alpha$ ' in Ni atoms, based on the Kyoto-Munich $K^{-} N$ chiral model amplitude (KM in Fig. 1) augmented by a phenomenological amplitude $B\left(\rho / \rho_{0}\right)^{\alpha}$ with three values of exponent $\alpha$, all providing good global fits to kaonic atoms data and satisfying the SNAF experimental constraint marked by horizontal dashed lines in Fig. 4. Also shown is a purely phenomenological optical potential producing a similarly good fit to kaonic atom data. The real parts of the four plotted potentials agree with each other up to about $25 \%$ of the central nuclear density. In contrast, the imaginary part is seen to be well determined up to $50 \%$ of the central density, reflecting the observation that strong interaction effects in kaonic atoms, where 

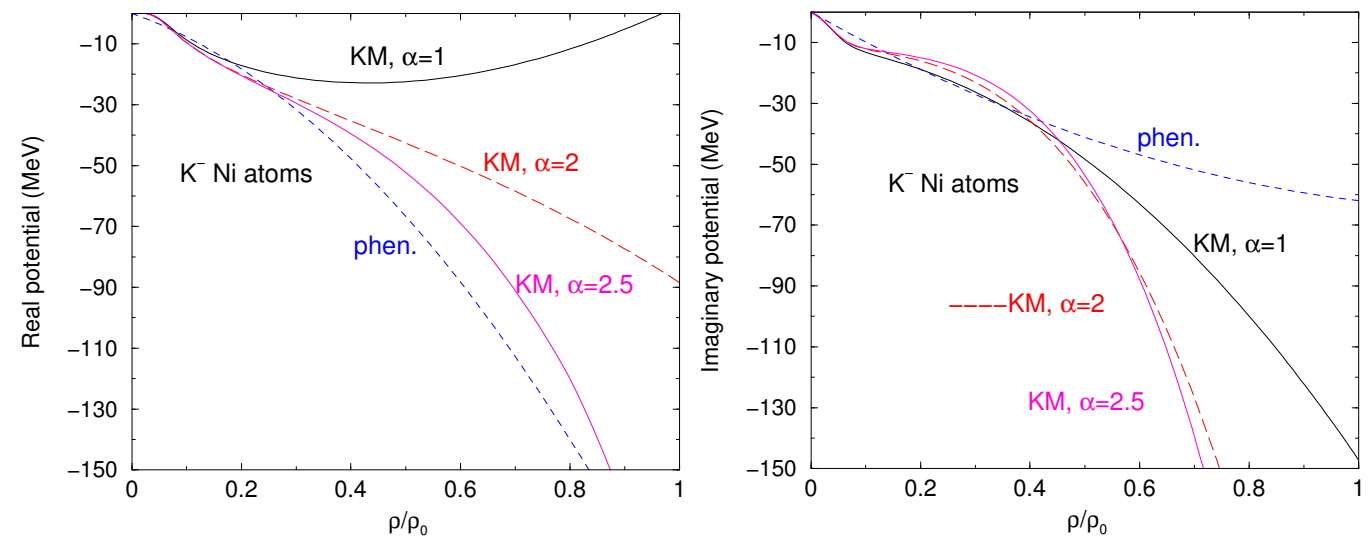

Figure 5: Real part (left) and imaginary part (right) of best-fit $K^{-} \mathrm{Ni}$ optical potentials ' $\mathrm{KM} \alpha$ ' based on the KM $1 N$ amplitude plus a phenomenological $m N$ amplitude $B\left(\rho / \rho_{0}\right)^{\alpha}$. Shown for comparison in shortdashed lines is a purely phenomenological potential. Figure adapted from Ref. [7].

level widths are significantly larger than shifts, are dominated by the imaginary potential. Hence we conclude that at larger densities the various models produce just analytic continuations from values close to the nuclear surface. Note that the three extrapolated imaginary $\mathrm{KM} \alpha$ potentials are deeper than the purely phenomenological imaginary potential. It is reassuring that the SNAF are largely determined by $\operatorname{Im} V_{K^{-}}$contributions from densities $10-20 \%$ of $\rho_{0}$ where the balance between $V_{K^{-}}^{1 N}$ and $V_{K^{-}}^{m N}$ is unambiguously related to the $1 N$ model used.
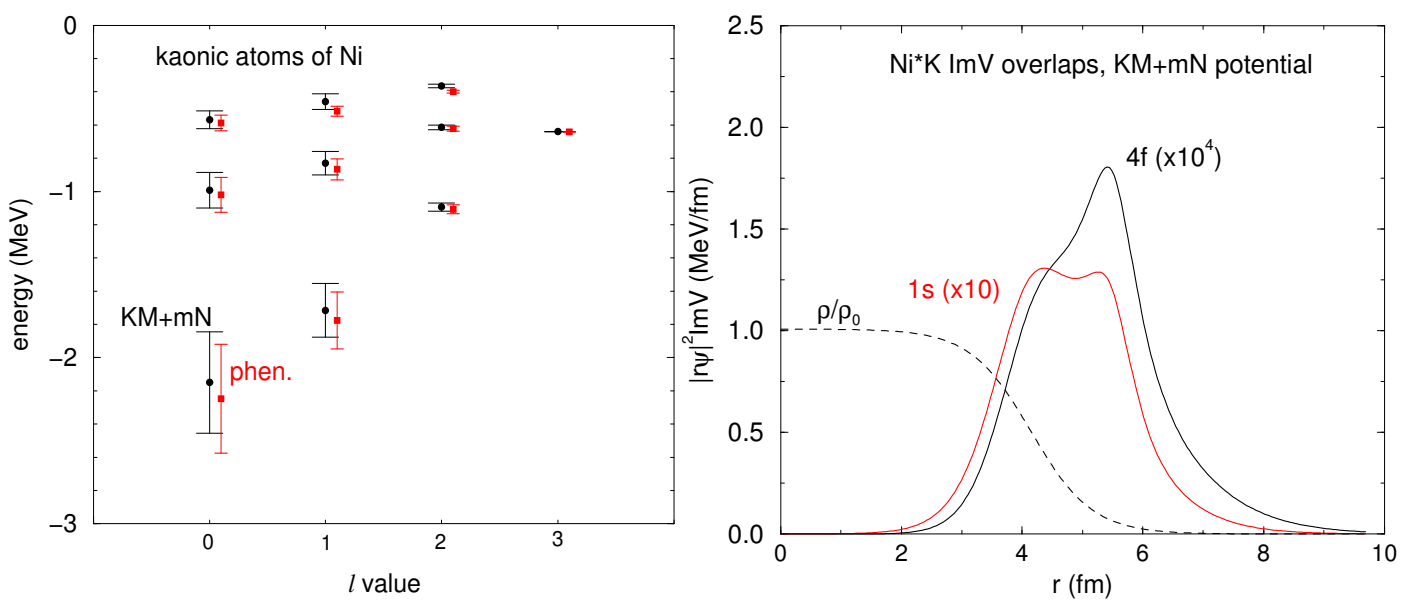

Figure 6: Left: energy levels and widths of kaonic atoms of Ni, calculated (in red) for a purely phenomenological best-fit potential $[13,14]$ and (in black) for the KM1 optical potential. Right: Overlaps of the absolute value squared of the $4 \mathrm{f}$ and $1 \mathrm{~s}$ radial wave function in kaonic atoms of $\mathrm{Ni}$ with the imaginary part of the KM1 optical potential. Shown in dash is the relative nuclear density of Ni.

Fig. 6 shows spectra (left panel) and overlaps (right panel) from Eq. (2.1) for kaonic atoms of Ni. The positions, as well as widths of energy levels plotted for two optical potentials (see caption) nearly coincide, provided these potentials fit the entire data on kaonic atoms. In particular the 
width of the lowest 1s state is rather small, less than $1 \mathrm{MeV}$, in spite of the large nuclear surface values of $\operatorname{Im} V_{K^{-}} \sim-50 \mathrm{MeV}$ from Fig. 5. The resolution of this apparent paradox is that this same strongly absorptive $\operatorname{Im} V_{K^{-}}$acts as inner repulsion, excluding the $K^{-}$meson in its $1 \mathrm{~s}$ atomic state from penetrating the nucleus nearly as much as it does in the X-ray observed $4 \mathrm{f}$ state, as shown on the right panel. It therefore appears that deeply bound kaonic atom states [13, 14], if ever measured in strong-interaction production reactions, will not provide information on the interior different from that known from normal X-ray states.

\section{3. $K^{-}$nuclear quasibound states}
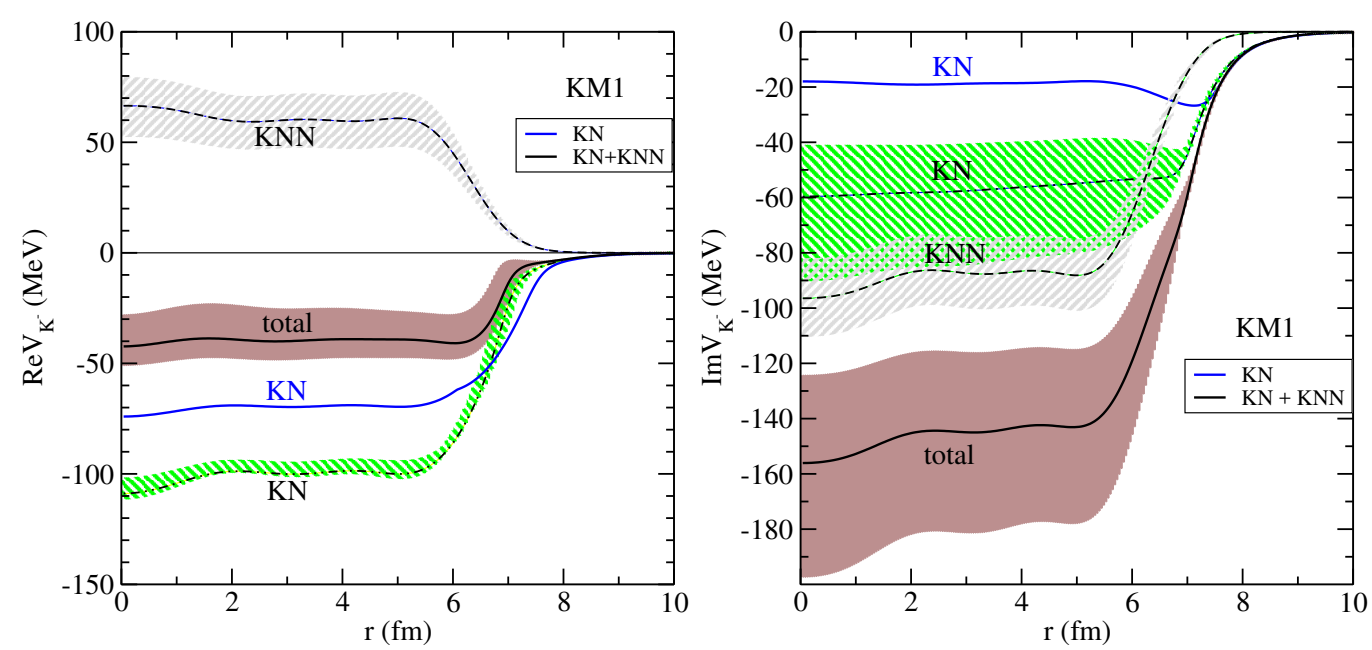

Figure 7: Contributions from $K^{-} N$ (dash-dotted) and $K^{-} N N$ (dashed) terms to the total real (left) and imaginary (right) $K^{-}$optical potential KM1 for strongly bound $K^{-}$states calculated self consistently in ${ }^{208} \mathrm{~Pb}$. Shaded areas denote uncertainty bands. Switching off $V_{K^{-}}^{m N}$, the purely-KM $V_{K^{-}}^{1 N}$ optical potential is shown for comparison ( $K N$, blue solid lines). Figure adapted from Ref. [9].

In contrast to $K^{-}$atomic states, wavefunctions of $K^{-}$nuclear states are fully confined within the nucleus. The dominance of the $m N$ component of $V_{K^{-}}$, particularly its imaginary part as noted for kaonic atoms in Sect. 2 is also realized in most of the models considered in Refs. [8,9] discussing $K^{-}$nuclear quasibound states. This is demonstrated for the KM1 optical potential in Fig. 7. Recall that of the six model amplitudes of Fig. 1, P and KM are the only ones passing the test of producing realistic values of SNAF [7]. The $1 N$ (denoted $K N)$ and $m N($ denoted $K N N$ ) potential contributions calculated self consistently in ${ }^{208} \mathrm{~Pb}$, including their uncertainties, are plotted in this figure as a function of the radial distance from the nuclear center. The $1 N$ component of $V_{K^{-}}$is seen to differ from the input $1 N$ term (blue solid lines) owing to the different subthreshold energy shifts obtained upon in(ex)cluding the $m N$ phenomenological term. The marked uncertainties reflect the kaonic-atom fit uncertainties in the values of the $m N$ strength parameter $B$. Whereas the resulting real potential depths are lower than obtained in totally phenomenological analyses [12], the imaginary potentials that are dominated by the $m N$ component are extremely deep, close to $160 \mathrm{MeV}$ (and much higher in the KM2 optical potential). Consequently, $K^{-}$nuclear quasibound 
states calculated in these KM-based, as well as P-based optical potentials are also extremely broad, with widths exceeding $100 \mathrm{MeV}$ each. This conclusion holds across the periodic table, from ${ }^{6} \mathrm{Li}$ to ${ }^{208} \mathrm{~Pb}$ [9], leaving room for observation of $K^{-}$nuclear quasibound states only in the lightest systems such as $K^{-} p p$.

\section{Conclusion}

Six chirally-inspired $K^{-} N$ interaction models [4] were used in global fits to kaonic atoms [7]. As expected, they all require additional purely phenomenological multi-nucleon terms to provide state-of-the-art fits. Although appearing equivalent on this basis, only two models (P and KM) pass the test of reproducing the SNAF extracted in bubble-chamber experiments. It was found that the derived $K^{-}$optical potentials are meaningful only for densities up to $25 \%$ of $\rho_{0}$ for the real part and up to $50 \%$ of $\rho_{0}$ for the imaginary part. Revisiting deeply bound kaonic atoms $[13,14]$ it was found that such states are well defined, but are hardly sensitive to details of best-fit optical potentials. This is caused by the poor overlap of $K^{-}$atom wave functions with the nucleus, which limits the kaon to relatively small nuclear densities and also makes these states remarkably narrow.

For $K^{-}$nuclear quasibound states [8,9] in contrast, the overlap of $K^{-}$nuclear wavefunctions with the nucleus is substantial. Hence, the widths calculated for these states exceed $100 \mathrm{MeV}$ when the optical potential $V_{K^{-}}$is extrapolated beyond its range of applicability of about $0.5 \rho_{0}$. We conclude that a search for such states in nuclei heavier than ${ }^{4} \mathrm{He}$ appears hopeless.

\section{Acknowledgments}

This work was partly supported by the GACR Grant No. P203/15/04301S.

\section{References}

[1] V. Metag, M. Nanova, E.Ya. Paryev, Prog. Part. Nucl. Phys. 97 (2017) 199.

[2] C.J. Batty, E. Friedman, A. Gal, Phys. Rep. 287 (1997) 385.

[3] E. Friedman, A. Gal, Phys. Rep. 452 (2007) 89.

[4] A. Cieplý, M. Mai, U.-G. Meißner, J. Smejkal, Nucl. Phys. A 954 (2016) 17.

[5] A. Cieplý, E. Friedman, A. Gal, D. Gazda, J. Mareš, Phys. Lett. B 702 (2011) 402.

[6] A. Cieplý, E. Friedman, A. Gal, D. Gazda, J. Mareš, Phys. Rev. C 84 (2011) 045206.

[7] E. Friedman, A. Gal, Nucl. Phys. A 959 (2017) 66.

[8] J. Hrtánková, J. Mareš, Phys. Lett. B 770 (2017) 342.

[9] J. Hrtánková, J. Mareš, Phys. Rev. C 96 (2017) 015205.

[10] T. Waas, M. Rho, W. Weise, Nucl. Phys. A 617 (1997) 449.

[11] E. Friedman, A. Gal, Nucl. Phys. A 928 (2014) 128.

[12] J. Mareš, E. Friedman, A. Gal, Nucl. Phys. A 770 (2006) 84.

[13] E. Friedman, A. Gal, Phys. Lett. B 459 (1999) 43.

[14] E. Friedman, A. Gal, Nucl. Phys. A 658 (1999) 345. 